\section{Surgery for lung herniation: a new approach for an old problem}

\author{
Marco Scarci, ${ }^{1}$ Andrea Billè, ${ }^{2}$ \\ Imran Zahid, ${ }^{3}$ Tom Routledge ${ }^{2}$ \\ 'Department of Thoracic Surgery, \\ St Joseph's Healthcare, McMaster \\ University, Hamilton, Ontario, Canada; \\ 2Department of Thoracic Surgery, Guy's \\ Hospital; ${ }^{3}$ Imperial College Medical \\ School, London, UK
}

\begin{abstract}
Lung herniation is frequently related to previous chest surgery or thoracic trauma. It can be symptomatic. Surgery with new titanium rib prosthesis is an interesting device to repair the chest wall in order to obtain better cosmetic and physiological results.
\end{abstract}

\section{Introduction}

Intercostal pulmonary herniation is a rare phenomenon and is defined as a protrusion of the lung beyond the normal confines of the thoracic cavity. We report the case of a 60 -yearold gentleman treated with a novel approach comprising moldable titanium clips and biological patch in order to obtain better physiological and cosmetic results.

\section{Case Report}

A 60-year-old gentleman was referred to us for a persisting bulge in the anterior portion of the left lower chest. He complained of pain on movements and shortness of breath. Past medical history includes ankylosing spondylitis.

Computed tomography (CT) of the chest excluded pathological rib fractures, and showed a defect of the anterior chest wall of $6.5 \mathrm{~cm}$ for $7.0 \mathrm{~cm}$. Radionuclide bone scan and myeloma screen were also negative. Dualenergy X-ray absorptiometry (DEXA-scan) also showed no evidence of osteoporosis. At surgery a large area of weakness was identified in the antero-lateral portion of the left lower chest (Figure 1). Although the patient denied recent chest trauma, the defect was bordered by two fractured ribs with signs of malunion. Those were repaired using a novel moldable titanium clip (STRACOS) to restore the chest wall continuity and provide further strength. The implants were crimpled on to the ribs with specifically designed instruments, avoiding compression of the intercostal bundle (Figure 2A). A biological patch (Veritas) was further laid on top of the repair to avoid recurrence (Figure 2B). The patient had excellent pain control from the procedure and was discharged home on regular paracetamol on day 6 . After one month he was further seen in the outpatients' clinic where he had no sign of recurrence and complete resolution of his symptoms.

\section{Discussion}

Pulmonary herniations can be congenital or acquired. Acquired hernias represent $80 \%$ of the total and $50 \%$ of them are the result of a trauma, but there are also spontaneous cases reported after maneuvers that increase the intrathoracic pressure. ${ }^{1}$

The antero-lateral portion of the thorax, between the $8^{\text {th }}$ and $9^{\text {th }}$ ribs is the site of predilection for spontaneous lung herniations, presumably because of the lack of muscular support provided to the posterior thoracic wall by the trapezius, latissimus dorsi, and rhomboid muscles. ${ }^{2,3}$

Treatment of this condition has been frequently conservative with compressive pads and corsets. This approach is often associated with problems represented by reduction of chest wall movement with consequent atelectasis and increased risk of infections. Surgical treatment is recommended in case of symptoms evocating incarceration, such as pain, hemoptysis, or recurrent infections and for cosmetic reasons or if it interferes with everyday activities. Traditionally pericostal fixation of the adjacent ribs may be sufficient for bridging small defects. ${ }^{4}$ For larger defects, reconstructive procedures are recommended. To avoid the risk of recurrences synthetic materials, such as Marlex mesh or polytetrafluoroethylene (PTFE) patch, are laid over the repair. However the risk of infection is always present. In our case we showed the use of a new rigid system for chest-wall reconstruction, consisting in moldable titanium bars and rib clips (Strasbourg Thoracic 0steosyntheses System STRACOS; MedXpert GmbH, Heitersheim, Germany) used in combination with a biological patch (Veritas - Synovis Life Technologies, Inc.) for repair of lung herniations. This combination of technologies we believe has advantages over current techniques.

The use of malleable metal clip to restore anatomic rib continuity is intuitively likely to preserve the mechanics of ventilation better than a patch repair because the natural bucket-handle movement of the ribs is retained.

Our solution also improves cosmetic results, thanks to the moldable property of the titani-
Correspondence: Marco Scarci, Department of Thoracic Surgery, St Joseph's Healthcare, McMaster University, 50 Charlton Avenue East, L8N 4A6, Hamilton, Ontario, Canada.

Tel: +1.905.906.9131 - Fax: +1.905.540.6512.

E-mail: marco.scarci@mac.com

Key words: intercostal pulmonary hernia, surgical approach, mesh.

Marco Scarci and Andrea Billè contributed equally to this article and should be therefore both considered as first authors.

Received for publication: 19 June 2011.

Accepted for publication: 28 August 2011.

This work is licensed under a Creative Commons Attribution NonCommercial 3.0 License (CC BYNC 3.0).

(C) Copyright M. Scarci et al., 2011

Licensee PAGEPress, Italy

Surgical Techniques Development 2011; 1:e14

doi:10.4081/std.2011.e14

um, which can be adapted to different shapes

In our case we found at surgery a large soft tissue weakness above the ribs defect, the use of a flexible patch sutured over the framework of titanium clips or bars was, therefore, used to provide a base for overlying soft tissue recon-

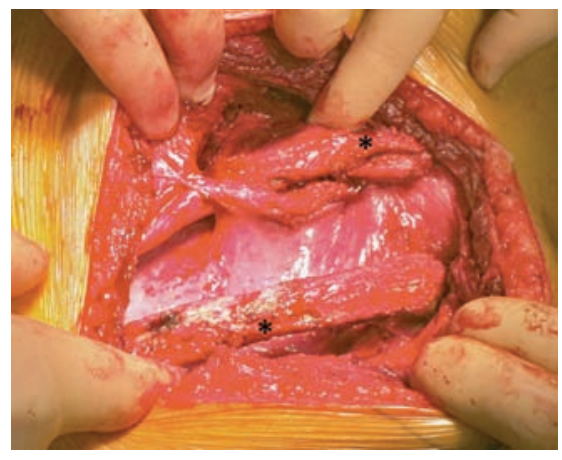

Figure 1. Intraoperative photo showing the defect.

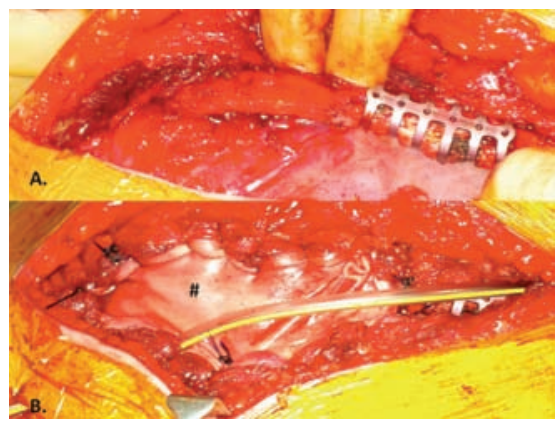

Figure 2. Veritas collagen patch laid over the repair. 
struction, and to prevent re-herniation between the bars. The patch was put over the titanium prosthesis to re-establish the continuity of the chest wall and to increase its stability creating a uniform layer between the fibrotic tissue after patch reabsorption and the muscles around the defect. Veritas pericardium is acellular, biocompatible with extremely low level of extractable DNA and has been used in closing large fascia defects in abdominal wall and breast surgical practice, particularly in the presence of established infection. The patch fosters remodeling by supporting both angiogenesis and cellular ingrowth. The result is the seamless integration of the implant into the surrounding tissue. Animal studies demonstrate that after as little as one month, implant is histologically indistinguishable from the host's native tissue. Avoiding the traditional intercostal sutures technique also allows reducing the risk of ischemia to the surrounding tissue due to compression of intercostal vessels and soft tissues. This proved, in our experience, to be particularly valuable in large defect where the amount of soft tissue dissection and diathermy usage to achieve hemostasis is considerable.

In conclusion, we recommend early surgical repair for all intercostal lung herniations because surgery offers the best results, with low morbidity and no mortality reported to date. The combination of rib prosthesis and patch get better cosmetic and physiological results: the titanium rib prosthesis guarantee the physiological shape and the physiological movements of the chest wall, and the Veritas patch is helpful in recreating the chest wall stability reducing the risk of infection, because it is quickly replaced by native tissue.

\section{References}

1. Forty J, Wells FC. Traumatic intercostal pulmonal hernia. Ann Thorac Surg 1990; 49:670 1.

2. Rusca M, Carbognani P, Cattelani L, et al. Spontaneous intercostal pulmonary hernia. J Cardiovasc Surg 2000;41:641-2.

3. Goverde P, Van Schil P, Van Den Brande F, Vanmaele R. Chronic herniation of the lung in a patient with chronic pulmonary disease. Case report and review of the literature. Thorac Cardiovasc Surg 1998;46: 164-6.

4. Balkan MF, Karam M, Oktar GL, Ünlü E. Transdiaphragmatic intercostal hernia following a penetrating thoracoabdominal injury: report of a case. Surg Today 2001;31: 708-11. 